\title{
Association of LINC00673 rs11655237 polymorphism with pediatric glioma susceptibility in a Chinese population
}

\author{
Zhuorong Zhang ${ }^{1 \#}$, Yihuan Huang ${ }^{1 \#}$, Ping Wu ${ }^{1 \#}$, Honghao Chen ${ }^{1}, Z_{\text {Zhijian Deng }}{ }^{1}$ Gaoyan Deng ${ }^{1}$, \\ Yongqin Zheng ${ }^{1}$, Guoyuan $\mathrm{Li}^{1}$, Li Yuan ${ }^{2}, \mathrm{Le} \mathrm{Li}^{3}$ \\ ${ }^{1}$ Department of Comprehensive and Emergency Surgery, Guangzhou Women and Children's Medical Center, Guangzhou Medical University, \\ Guangzhou, China; ${ }^{2}$ Department of Pathology, Guangzhou Women and Children's Medical Center, Guangzhou Medical University, Guangzhou, \\ China; ${ }^{3}$ Department of Thoracic Surgery, Guangzhou Women and Children's Medical Center, Guangzhou Medical University, Guangzhou, China \\ Contributions: (I) Conception and design: L Li, Z Deng, L Yuan; (II) Administrative support: None; (III) Provision of study materials or patients: \\ None; (IV) Collection and assembly of data: Z Zhang, P Wu, H Chen, Y Zheng, G Li; (V) Data analysis and interpretation: L Li, Y Huang; (VI) \\ Manuscript writing: All authors; (VII) Final approval of manuscript: All authors. \\ \#These authors contributed equally to this work. \\ Correspondence to: Li Le, PhD, MD. Department of Thoracic Surgery, Guangzhou Women and Children's Medical Center, Guangzhou Medical \\ University, 9 Jinsui Road, Guangzhou 510623, China. Email: 18922103361@126.com; Yuan Li, PhD, MD. Department of Pathology, Guangzhou \\ Women and Children's Medical Center, Guangzhou Medical University, 9 Jinsui Road, Guangzhou 510623, China. Email: lizzyklarck@126.com.
}

Background: Previous researches have suggested that LINC00673 rs11655237 C>T polymorphism might be correlated to cancer susceptibility. However, its correlation with pediatric glioma is unknown. Therefore, this study aimed to determine whether LINC00673 rs11655237 C>T polymorphism is correlated with pediatric glioma.

Methods: In total, we included 399 subjects from South China. The Student's $t$-test was performed to evaluate age differences between glioma cases and controls. Differences in the categorical variables between the two groups were assessed using the $\chi^{2}$ test. A logistic regression was conducted to calculate the odds ratio (OR) and the $95 \%$ confidence interval (CI).

Results: We conducted this case-control study to investigate the association between LINC00673 polymorphism and pediatric glioma susceptibility. Our results revealed that LINC00673 rs11655237 C>T polymorphism was not correlated to pediatric glioma susceptibility in a Chinese population (CC/CT compared with TT: adjusted OR =2.49, 95\% CI: 0.87-7.15, P=0.091). Furthermore, a stratified analysis also indicated LINC00673 rs11655237 C>T polymorphism did not increase the risk of glioma in different subgroups.

Conclusions: Our study revealed that LINC00673 rs11655237 C>T polymorphism was not correlated to pediatric glioma susceptibility in a Chinese population. In the future, further exploration of this genetic factor in relation to glioma susceptibility will require a larger sample size to verify the current findings.

Keywords: LINC00673; polymorphism; glioma; susceptibility

Submitted Jun 04, 2021. Accepted for publication Jul 14, 2021.

doi: $10.21037 /$ tp-21-291

View this article at: https://dx.doi.org/10.21037/tp-21-291

\section{Introduction}

With the exception of leukemia, central nervous system (CNS) tumors are the most common tumors in children and adolescents. CNS tumors are difficult to treat, have a poor prognosis, and a high recurrence rate (1). With the rapid expansion in imaging technology, the diagnostic rate of new CNS tumors is increasing yearly. According to the latest statistics of United States, approximately 23,880 glioma cases are diagnosed, and 16,830 estimated new cases will die 
from glioma (2). Despite recent advances in treatment, the prognosis for glioma patients remains poor, and the average overall survival (OS) for glioblastoma patients is less than 16 months (3-5). Therefore, there is an urgent need to explore the molecular pathological mechanisms and potential molecular biomarkers for glioma.

Long noncoding RNAs (lncRNAs) are a type of complex molecule with little or no protein-coding ability and are transcribed in most eukaryotic genomes. Initially, lncRNAs were considered to be merely transcriptional noise without any biological function (6). However, there is growing evidence that LncRNAs regulate gene expression at the epigenetic, transcriptional, and post-transcriptional processing levels by acting on nucleic acid molecules and proteins and play a key role in many of the biological processes associated with tumors (7). Of these, LINC00673 is among the most thoroughly studied (8) and is located on chromosome $17 \mathrm{q} 24.3$, at approximately $275-\mathrm{kb}$ telomeric of SOX9. Analysis of this lncRNA sequence has shown that it possesses an absolutely conserved region, which is remarkably similar to the SRA1 protein. Therefore, this lncRNA is also known as "SRA-like non-coding RNA" (SLNCR) (9). Notably, Childs et al. first reported in a genome-wide association study (GWAS) of 9925 pancreatic cancer cases and 11,569 controls that the single nucleotide polymorphisms (SNPs) in LINC00673 were significantly associated with pancreatic cancer susceptibility in North America, Central Europe, and Australia (10). However, to date, the correlation between LINC00673 polymorphism and pediatric glioma susceptibility has not been investigated. Therefore, this study focused on whether LINC00673 polymorphism is associated with the risk of pediatric glioma by recruiting 399 subjects from South China. We present the following article in accordance with the STROBE reporting checklist (available at https://dx.doi.org/10.21037/ tp-21-291).

\section{Methods}

\section{Subjects}

In this case-control study, 399 subjects (171 cases and 228 controls) were included from South China as we reported before (Table S1) (11). All glioma patients were pathologically confirmed. We collected clinical information including patients' age, gender, and clinical stage. Before collecting blood samples, written informed consent was obtained from the parents or legal guardians of each child.
This investigation protocol was approved by the Ethics Committee of Guangzhou Women (No.: 2016021650) and Children's Medical Center. All procedures performed in this study involving human participants were in accordance with the Declaration of Helsinki (as revised in 2013).

\section{Polymorphism analysis}

LINC00673 rs11655237 C>T was selected based on previous publications $(12,13)$. Blood was collected in an EDTA test tube from 399 participants. Details of the blood draw, DNA extraction, and storage status have been described in a previous study (14). LINC00673 genotyping was conducted with TaqMan real-time quantitative PCR (QPCR). For quality control, the obtained variants of LINC00673 rs11655237 C>T polymorphism were confirmed by genotype in $10 \%$ of randomly selected genomic DNA samples, and the results of the quality control were in accord with the first assays.

\section{Statistical analysis}

Statistical analyses were performed with SAS software (version 9.4; SAS institute in United States). The HardyWeinberg equilibrium (HWE) of all SNPs in the controls was tested by the goodness-of-fit $\chi^{2}$ test. The Student's $t$-test was used to assess age differences between glioma cases and controls. Differences in categorical variables between the two groups were determined by the $\chi^{2}$ test. A multiple logistic regression model was used to calculate the odds ratio (OR) and the $95 \%$ confidence interval (CI). All $\mathrm{P}$ values were two-sided, and $\mathrm{P}<0.05$ was considered statistically significant.

\section{Results}

\section{Patient characteristics}

In this case-control study, to explore the correlation between LINC00673 rs1165237 C>T polymorphism and glioma susceptibility, we recruited 339 age- and gendermatched subjects (171 glioma cases and 228 non-tumor controls) from South China. The clinicopathological characteristics of all 339 subjects are listed in Table S1. The average ages of the glioma patients and controls were $63.40 \pm 47.72$ months (range, 4.00-168.00 months) and 52.41 \pm 32.65 months (range, 4.00-168.00 months), respectively. Overall, there were less females $(174,43.6 \%)$ 
Table 1 Association between LINC00673 rs11655237 C>T polymorphism and glioma risk

\begin{tabular}{|c|c|c|c|c|c|c|c|}
\hline Genotype & Cases $(\mathrm{N}=171)$ & Controls (N=228) & $\mathrm{P}^{\mathrm{a}}$ & Crude OR $(95 \% \mathrm{Cl})$ & $\mathrm{P}$ & Adjusted OR $(95 \% \mathrm{Cl})^{\mathrm{b}}$ & $\mathrm{P}^{\mathrm{b}}$ \\
\hline $\mathrm{CC}$ & $105(61.40)$ & $146(64.04)$ & & 1.00 & & 1.00 & \\
\hline CT & $56(32.75)$ & 76 (33.33) & & $1.03(0.67-1.57)$ & 0.911 & $0.98(0.64-1.52)$ & 0.941 \\
\hline $\mathrm{TT}$ & $10(5.85)$ & $6(2.63)$ & & $2.32(0.82-6.58)$ & 0.114 & $2.49(0.87-7.15)$ & 0.091 \\
\hline Dominant & $66(38.60)$ & $82(35.96)$ & 0.590 & $1.12(0.74-1.69)$ & 0.590 & 1.09 (0.72-1.65) & 0.683 \\
\hline Recessive & $161(94.15)$ & $222(97.37)$ & 0.105 & $2.30(0.82-6.45)$ & 0.114 & $2.50(0.88-7.12)$ & 0.086 \\
\hline
\end{tabular}

${ }^{a}, \chi^{2}$ test for genotype distributions between glioma patients and cancer-free controls. ${ }^{\mathrm{b}}$, adjusted for age and gender. OR, odds ratio; $\mathrm{Cl}$, confidence interval; HWE, Hardy-Weinberg equilibrium.

than males (225, 56.4\%). No significant differences in age and gender were identified between the case and control groups $(\mathrm{P}=0.623$ and $\mathrm{P}=0.190$, respectively). In the case group, patients with $\mathrm{WHO}$ grade I-II were in the majority (131, 76.61\%), with only 40 patients classified with high grade gliomas (23.39\%) (Table S1).

\section{Association of LNIC00673 rs11655237 C>T variants with pediatric glioma susceptibility}

All 399 subjects underwent genotype testing for LINC006773 rs11655237 C>T polymorphism. The genotype frequencies and percentage of LINC00673 rs11655237 C>T polymorphism are summarized in Table 1 . All genotypes were checked for quality control and were consistent with HWK in control subjects $(\mathrm{P}=0.289)$. Next, we investigated the association of LINC006773 rs11655237 C>T polymorphism and glioma susceptibility. LINC00673 rs11655237 C>T polymorphism showed no correlation with glioma susceptibility (CC vs. TT: adjusted OR $=2.49,95 \%$ CI: $0.87-7.15, \mathrm{P}=0.091$ ). A possible reason for this finding is a lack of statistical power due to our small sample size.

\section{Association of LINC00673 rs11655237 C>T variants with glioma susceptibility in different subgroups}

To identify whether LINC00673 rs11655237 C>T variants were correlated with the risk of glioma in different subgroups, we performed a stratified analysis of glioma patients based on their clinicopathological characteristics after adjustments for age and gender. Table 2 shows the genotype frequencies of LINC00673 rs11655237 C>T polymorphism in the different subgroups. The association of LINC00673 rs11655237 C>T polymorphism and the risk of glioma did not reach statistical significance for any of the subgroups (Table 2). Therefore, in the present study, LINC00673 rs11655237 C>T polymorphism did not demonstrate a significant correlation with pediatric glioma susceptibility in a Chinese population.

\section{Discussion}

A growing body of evidence shows that heredity plays a key role in cancer susceptibility. Over the past decade, scientists have published more than 1,300 GWAS, and identified nearly 6,500 gene loci associated with diseases (15). However, just $7 \%$ of these sites were located in proteincoding regions, while the remaining $93 \%$ were located mainly in non-coding regions. Presently, four different variants of SNPs have been identified: transformation, transposition, deletion, and insertion. Gene mutations in protein-coding regions often cause harmful or fatal outcomes, making it impossible for carriers to survive long enough for cancer to develop (16). However, mutations in regulated regions may cause small changes in the gene expression in cell types or in tissue-specific ways that may confer cancer susceptibility in the carrier $(17,18)$. Currently, many SNPs have been correlated with cancer susceptibility, such as in gastric cancer, lung cancer, and other malignant tumors $(19,20)$.

We conducted this case-control study to investigate the association between LINC00673 polymorphism and pediatric glioma susceptibility. Our results suggested that LINC00673 rs11655237 C>T polymorphism was not correlated with glioma susceptibility in a pediatric Chinese population, which is inconsistent with results 
Table 2 Stratification analysis between LINC00673 rs11655237 C>T polymorphism and glioma risk

\begin{tabular}{|c|c|c|c|c|c|c|}
\hline Variables & \multicolumn{2}{|c|}{ rs1042522 (cases/controls) } & $\frac{\text { Crude OR }}{(95 \% \mathrm{Cl})}$ & $\mathrm{P}$ & $\frac{\text { Adjusted OR }}{\text { a }}$ & $P^{a}$ \\
\hline \multicolumn{7}{|l|}{ Age, months } \\
\hline$<60$ & $53 / 76$ & $33 / 43$ & $1.12(0.63-1.99)$ & 0.695 & $1.12(0.63-1.98)$ & 0.710 \\
\hline$\geq 60$ & $53 / 70$ & $33 / 39$ & $1.12(0.63-2.01)$ & 0.710 & $1.10(0.61-1.99)$ & 0.743 \\
\hline Females & $47 / 59$ & $34 / 34$ & $1.26(0.68-2.31)$ & 0.465 & $1.23(0.66-2.29)$ & 0.511 \\
\hline Males & $58 / 87$ & $32 / 48$ & $1.00(0.57-1.75)$ & 1.000 & $0.99(0.56-1.73)$ & 0.963 \\
\hline \multicolumn{7}{|l|}{ Subtype } \\
\hline Astrocytic tumors & $75 / 146$ & $50 / 82$ & $1.19(0.76-1.86)$ & 0.454 & $1.16(0.73-1.83)$ & 0.536 \\
\hline Embryonal tumors & $5 / 146$ & $2 / 82$ & $0.71(0.14-3.75)$ & 0.689 & $0.61(0.10-3.83)$ & 0.595 \\
\hline \multicolumn{7}{|l|}{ Clinical stages } \\
\hline I & $59 / 146$ & $44 / 82$ & $1.33(0.83-2.14)$ & 0.242 & $1.33(0.82-2.15)$ & 0.248 \\
\hline II & $17 / 146$ & $11 / 82$ & $1.15(0.52-2.58)$ & 0.730 & $1.15(0.52-2.59)$ & 0.727 \\
\hline III & $11 / 146$ & $4 / 82$ & $0.65(0.20-2.10)$ & 0.469 & $0.66(0.20-2.17)$ & 0.497 \\
\hline IV & $18 / 146$ & $7 / 82$ & $0.69(0.28-1.73)$ & 0.431 & $0.66(0.25-1.75)$ & 0.403 \\
\hline$I+I I$ & $76 / 146$ & $55 / 82$ & $1.29(0.83-2.00)$ & 0.259 & $1.27(0.81-1.97)$ & 0.296 \\
\hline III+IV & $29 / 146$ & $11 / 82$ & $0.68(0.32-1.42)$ & 0.302 & $0.66(0.31-1.40)$ & 0.276 \\
\hline
\end{tabular}

${ }^{\mathrm{a}}$, adjusted for age and gender, omitting the corresponding stratify factor. OR, odds ratio; $\mathrm{Cl}$, confidence interval.

found in other solid tumors. This different outcome may be related to variations in sample populations, differing research protocols, and the small sample size in the present study. In other solid tumors, current research has focused on the association of the SNPs of LINC00673 (rs11655237 C>T polymorphism) and cancer risk. Zheng et al. reported that rs11655237 is located on exon 4 of LINC00673 and six SNPs are involved in the highlinkage disequilibrium with rs11655237 (21). LINC00673 rs11655237 C>T polymorphism may increase the risk of many solid cancers in an allele-specific manner $(12,21,22)$. One possible reason is that rs 11655237 could be recognized and complemented with the miR-1231 domain, which further regulates the expression level of LINC00673 (23). In fact, the formation of this miR-1231-recognition region is caused by the single-base substitution of the $G$ allele to the A allele at rs11655237, and miR-1231 may bind to the recognition region and further reduce the expression level of LINC00673. Previous studies have reported that LINC00673 acts as a tumor suppressor gene in a variety of tumors, and downregulation of LINC00673 could increase the risk of tumor susceptibility in subjects $(21,24)$. Zhu et al. (24) found that cervical cancer patients with the A allele of rs116552337 had a poorer prognosis than those with the $\mathrm{G}$ allele of rs11655237. These findings suggest that LINC00673 plays an important role in maintaining cell homeostasis, and its polymorphisms could increase the risk of cancer susceptibility. However, how SNPs affect LINC00673 binding to transcription factors remains unclear.

This case-control study has several limitations. Firstly, the sample size was not large enough to obtain statistically significant results. Thus, a larger sample size is needed in future studies to verify the above results. Secondly, a much higher number of SNP loci should be considered in any further research. We cannot rule out the influence of other 
loci polymorphisms on pediatric glioma susceptibility. Thirdly, more studies are needed to consider the role of other potentially confounding variables, such as environmental factors.

In conclusion, as far as we know, this is the first casecontrol study focusing on the possible association of LINC00673 rs11655237 C>T polymorphism and glioma risk. In contrast to other LINC00673 rs11655237 C>T polymorphism studies, we found that LINC00673 rs11655237 C>T polymorphism was not correlated with pediatric glioma susceptibility in a Chinese population.

\section{Acknowledgments}

Funding: This study was supported by grants from the Guangzhou Science and Technology Planning Project (No: 202002030007).

\section{Footnote}

Reporting Checklist: The authors have completed the STROBE reporting checklist. Available at https://dx.doi. org/10.21037/tp-21-291

Data Sharing Statement: Available at https://dx.doi. org/10.21037/tp-21-291

Conflicts of Interest: All authors have completed the ICMJE uniform disclosure form (available at https://dx.doi. org/10.21037/tp-21-291). The authors have no conflicts of interest to declare.

Ethical Statement: The authors are accountable for all aspects of the work in ensuring that questions related to the accuracy or integrity of any part of the work are appropriately investigated and resolved. All procedures performed in this study involving human participants were in accordance with the Declaration of Helsinki (as revised in 2013). The study was approved by the Ethics Committee of Guangzhou Women (No. 2016021650) and Children's Medical Center and informed consent was taken from all the patients.

Open Access Statement: This is an Open Access article distributed in accordance with the Creative Commons Attribution-NonCommercial-NoDerivs 4.0 International License (CC BY-NC-ND 4.0), which permits the noncommercial replication and distribution of the article with the strict proviso that no changes or edits are made and the original work is properly cited (including links to both the formal publication through the relevant DOI and the license). See: https://creativecommons.org/licenses/by-nc-nd/4.0/.

\section{References}

1. Ostrom QT, Gittleman H, Truitt G, et al. CBTRUS Statistical Report: Primary Brain and Other Central Nervous System Tumors Diagnosed in the United States in 2011-2015. Neuro Oncol 2018;20:iv1-86.

2. Siegel RL, Miller KD, Jemal A, et al. Cancer statistics, 2018. CA Cancer J Clin 2018;68:7-30.

3. Fan CD, Fu XY, Zhang ZY, et al. Selenocysteine induces apoptosis in human glioma cells: evidence for TrxR1targeted inhibition and signaling crosstalk. Sci Rep 2017;7:6465.

4. Gomez GA, Oksdath M, Brown MP, et al. New approaches to model glioblastoma in vitro using brain organoids: implications for precision oncology. Transl Cancer Res 2019;8:S606-11.

5. Qu S, Chen Z, Liu B, et al. N6-methyladenine-related genes affect biological behavior and the prognosis of glioma. Cancer Med 2021;10:98-108.

6. Jing L, Gong M, Lu X, et al. LINC01127 promotes the development of ovarian tumors by regulating the cell cycle. Am J Transl Res 2019;11:406-17.

7. Chen Y, Zitello E, Guo R, et al. The function of LncRNAs and their role in the prediction, diagnosis, and prognosis of lung cancer. Clin Transl Med 2021;11:e367.

8. Li N, Cui Z, Huang D, et al. Association of LINC00673 rs11655237 polymorphism with cancer susceptibility: A meta-analysis based on 23,478 subjects. Genomics 2020;112:4148-54.

9. Zhu K, Gong Z, Li P, et al. A review of linc00673 as a novel lncRNA for tumor regulation. Int J Med Sci 2021:18:398-405.

10. Childs EJ, Mocci E, Campa D, et al. Common variation at $2 \mathrm{p} 13.3,3 \mathrm{q} 29,7 \mathrm{p} 13$ and $17 \mathrm{q} 25.1$ associated with susceptibility to pancreatic cancer. Nat Genet 2015;47:911-6.

11. He J, Li Y, Lin HR, et al. Genetic variants in $\mathrm{m} 6 \mathrm{~A}$ modification core genes are associated with glioma risk in Chinese children. Mol Ther Oncolytics. 2021 ;20:199-208.

12. Li Y, Zhuo ZJ, Zhou H, et al. Additional data support the role of LINC00673 rs11655237 C>T in the development of neuroblastoma. Aging (Albany NY) 2019;11:2369-77.

13. Zhang Z, Chang Y, Jia W, et al. LINC00673 rs11655237 
C> T confers neuroblastoma susceptibility in Chinese population. Biosci Rep 2018;38:BSR20171667.

14. He J, Zhang R, Zou Y, et al. Evaluation of GWASidentified SNPs at 6p22 with neuroblastoma susceptibility in a Chinese population. Tumour Biol 2016;37:1635-9.

15. Visscher PM, Wray NR, Zhang Q, et al. 10 Years of GWAS Discovery: Biology, Function, and Translation. Am J Hum Genet 2017;101:5-22.

16. Collins FS, Brooks LD, Chakravarti A, et al. A DNA polymorphism discovery resource for research on human genetic variation. Genome Res 1998;8:1229-31.

17. Zhang X, Li J, Yang Q, et al. Tumor mutation burden and JARID2 gene alteration are associated with short diseasefree survival in locally advanced triple-negative breast cancer. Ann Transl Med 2020;8:1052.

18. Castellanos-Rubio A, Ghosh S, et al. Disease-Associated SNPs in Inflammation-Related lncRNAs. Front Immunol 2019; 10:420.

19. Sung H, Yang HH, Hu N, et al. Functional annotation of high-quality SNP biomarkers of gastric cancer susceptibility: the Yin Yang of PSCA rs2294008. Gut 2016;65:361-4.

Cite this article as: Zhang Z, Huang $\mathrm{Y}, \mathrm{Wu} \mathrm{P}$, Chen $\mathrm{H}$, Deng Z, Deng G, Zheng Y, Li G, Yuan L, Li L. Association of LINC00673 rs11655237 polymorphism with pediatric glioma susceptibility in a Chinese population. Transl Pediatr 2021;10(7):1890-1895. doi: 10.21037/tp-21-291
20. O'Brien TD, Jia P, Caporaso NE, et al. Weak sharing of genetic association signals in three lung cancer subtypes: evidence at the SNP, gene, regulation, and pathway levels. Genome Med 2018;10:16.

21. Zheng J, Huang $X$, Tan $W$, et al. Pancreatic cancer risk variant in LINC00673 creates a miR-1231 binding site and interferes with PTPN11 degradation. Nat Genet 2016;48:747-57.

22. Zhao K, Zhang R, Li T, et al. Functional variants of lncRNA LINC00673 and gastric cancer susceptibility: a case-control study in a Chinese population. Cancer Manag Res 2019;11:3861-8.

23. Wang Y, Luo T, et al. LINC00673 rs11655237 Polymorphism Is Associated With Increased Risk of Cervical Cancer in a Chinese Population. Cancer Control 2018;25:1073274818803942.

24. Zhu W, Li L, Li D, et al. Rs11655237 polymorphism of LINC00673 affects the prognosis of cervical cancer by interfering with the interaction between LINC00673 and microRNA-1231. J Cell Physiol 2020;235:8155-66.

(English Language Editor: D. Fitzgerald) 


\section{Supplementary}

Table S1 Frequency distribution of selected variables in glioma patients and cancer-free controls

\begin{tabular}{|c|c|c|c|c|c|}
\hline Variables & \multicolumn{2}{|c|}{ Cases $(\mathrm{N}=171)$} & \multicolumn{2}{|c|}{ Controls $(\mathrm{N}=228)$} & $P^{a}$ \\
\hline Age range, months & \multicolumn{2}{|c|}{$4.00-168.00$} & \multicolumn{2}{|c|}{$4.00-168.00$} & 0.623 \\
\hline Mean \pm SD & \multicolumn{2}{|c|}{$63.40 \pm 47.72$} & \multicolumn{2}{|c|}{$52.41 \pm 32.65$} & \\
\hline$<60$ & 85 & 49.71 & 119 & 52.19 & \\
\hline Gender & & & & & 0.190 \\
\hline Female & 81 & 47.37 & 93 & 40.79 & \\
\hline Male & 90 & 52.63 & 135 & 59.21 & \\
\hline \multicolumn{6}{|l|}{ Subtypes } \\
\hline Neuronal and mixed neutonal-glial tumours & 14 & 8.19 & - & - & \\
\hline Embryonal tumors & 7 & 4.09 & - & - & \\
\hline \multicolumn{6}{|l|}{ WHO stages } \\
\hline I & 103 & 60.23 & - & - & \\
\hline II & 28 & 16.37 & - & - & \\
\hline III & 15 & 8.77 & - & - & \\
\hline IV & 25 & 14.62 & - & - & \\
\hline
\end{tabular}

${ }^{\mathrm{a}}$, two-sided $\chi^{2}$ test for distributions between glioma patients and cancer-free controls. SD, standard deviation. 